\title{
Experimental and computational study on the bubble behavior in a 3-D fluidized bed
}

\author{
A. Acosta-Iborra*, C. Sobrino, F. Hernández-Jiménez, M. de Vega \\ Departamento de Ingeniería Térmica y de Fluidos, Universidad Carlos III de Madrid, ISE Research Group, Avda. de la Universidad 30, 28911 Leganés, Madrid, Spain
}

\begin{abstract}
A B S T R A C T
The results from a two fluid Eulerian Eulerian three dimensional (3 D) simulation of a cylindrical bed, filled with Geldart B particles and fluidized with air in the bubbling regime, are compared with experimental data obtained from pressure and optical probe measurements in a real bed of similar dimensions and operative conditions. The main objectives of this comparison are to test the validity of the simulation results and to characterize the bubble behavior and bed dynamics. The fluidized bed is $0.193 \mathrm{~m}$ internal diameter and $0.8 \mathrm{~m}$ height, and it is filled with silica sand particles, reaching a settle height of $0.22 \mathrm{~m}$. A frequency domain analysis of absolute and differential pressure signals in both the measured and the simulated cases shows that the same principal phenomena are reproduced with similar distributions of peak frequencies in the power spectral density (PSD) and width of the spectrum. The local dynamic behavior is also studied in the present work by means of the PSD of the simulated particle fraction and the PSD of the measured optical signal, which reveals as well good agreement between both the spectra. This work also presents, for the first time, comparative results of the measured and the simulated bubble size and velocity in a fully $3 \mathrm{D}$ bed configuration. The values of bubble pierced length and velocity retrieved from the experimental optical signals and from the simulated particle fraction compare fairly well in different radial and axial positions. Very similar values are obtained when these bubble parameters are deduced from either simulated pressure signals or simulated particle volume fraction. In addition, applying the maximum entropy method technique, bubble size probability density functions are also calculated. All these results indicate that the two fluid model is able to reproduce the essential dynamics and interaction between bubbles and dense phase in the $3 \mathrm{D}$ bed studied.
\end{abstract}

\section{Introduction}

Fluidized bed technology is widely used in process industry and energy production. Gas solid fluidized beds operating in the bubbling regime, for which high contact efficiency between the gaseous and the solid phases leads to high conversion and heat transfer rates, are now broadly commercialized. In this regime, the bubble flow is of main importance to obtain a good mixing between gaseous and solid phases, while the dynamic character istics of the fluidized bed, given by other properties such as pressure and pressure fluctuations, are relevant for the operation of the bed under stable conditions. Thus, both bubble flow and pressure dynamics can be considered major parameters during the design, operation and scale up of these systems. However, most of the work is still dependent on expensive pilot scale experiments along with empirical or semi empirical models obtained from

\footnotetext{
* Corresponding author: Tel.: +34 916248465.

E-mail address: aacosta@ing.uc3m.es (A. Acosta-Iborra).
}

laboratory studies. Therefore in the last years, modeling and numer ical simulations of fluidized beds have increased interest on them as a complementary tool to experiments.

Presently, simulation of small and medium scale gas fluidized beds is commonly undertaken by means of two fluid computational fluid dynamic (CFD) models, also known as Eulerian Eulerian two fluid models, which are primarily based on the representation of the gas phase and the particulate phase as two interpenetrating continua (Gidaspow, 1994; van Wachem and Almstedt, 2003). Two fluid models provide information about the macroscopic hydrodynamics (i.e. velocity and volume fraction) of the two phases, including the bubble formation and motion. Therefore, these models are especially suitable for the understanding of fluidized beds regarding dense phase bulk motion, and gas phase flow including bubbles. Although two fluid models have been applied in the literature with satisfactory results to predict the behavior of bubbles in fluidized beds, there are numerous questions that need further validation (Grace and Taghipour, 2004). For example, the closure equations for the particle drag, viscosity and pressure rely on the granular temperature theory, which is based on the assumption of 
isotropy in rapid granular flows (Gidaspow, 1994). The considera tion of particles as perfectly spherical is also a simplification that may deteriorate the accuracy of the simulated interparticle and interphase stresses. Besides, to make affordable the simulation, boundary conditions are simplifications of what actually occurs in beds. For example, the commonly employed boundary condition of uniform velocity (i.e. spatially homogeneous) for the air inlet at the bed distributor is a realistic assumption of beds under bubbling regime. However, small time fluctuations of the air entering the bed at the distributor might influence the bed dynamics as a result of the bed sensitivity to small perturbations (Peirano et al., 2002). A problem always present in fluidized bed simulations is the influence of the mesh resolution on the accuracy of the large gradients appearing at the bubble boundary. As bubbles can cross any point in the bed, the use of very fine meshes covering all the bed volume is unaffordable in three dimensional simulations. Owing to all these questions, there is a need of practical validation of two fluid models, which should be carried out for each particular bed geometry and regime.

A greater detail in the description of the particle phase can be obtained using Lagrangian models such as discrete particle models (Deen et al., 2007) and lattice Boltmann models (Ladd and Verberg, 2001), which allow the simulation of the individual motion of each particle. Although great progress has been done in the last few years in the field of Lagrangian models, their use is still restricted to a number of particles far below the amount encountered in fluidized beds of industrial interest.

Most of the comparisons between experiments and two fluid models presented in the literature account for two dimensional or quasi two dimensional (2 D) beds. van Wachem et al. (1998) compared with existing correlations the time averaged bubble size and velocity obtained with an Eulerian Eulerian multiphase CFD model of a 2 D square column filled with Geldart B particles in free bubbling regime. This study was completed in van Wachem et al. (1999), where the authors presented the dynamic characteristics of the gas solid behavior and compared it with published experimental data and correlations. The comparison included the velocity of pressure and voidage waves, the power spectra of pressure and voidage fluctuations and the Kolmogorov entropy, among other results. Taghipour et al. (2005) tested their model predictions of time averaged solid volume fraction, bed expansion ratio, pressure drop and qualitative gas solid flow pattern against experimentally obtained pressure drop data and local voidage calculations using a reflective optical fiber probe in a $2 \mathrm{D}$ Plexiglas column. The size distribution, rise velocity and visible flow of bubbles in a freely bubbling fluidized bed for Geldart B and D particles predicted by the constant viscosity model and the kinetic theory of granular flow models were compared by Patil et al. (2005) with correlations and experimen tal data taken from other authors. Wang et al. (2008) used a two fluid model in a $2 \mathrm{D}$ domain to study the flow behavior of particles in a riser; the computed results were compared with experimental particle distributions, velocities and bed expansion ratio measurements reported in literature for $2 \mathrm{D}$ systems. Passalacqua and Marmo (2009) performed a two fluid model simulation of a $2 \mathrm{D}$ bubbling fluidized bed with and without a central jet using different frictional stress models. They compared the equivalent diameter obtained from the area of their simulated bubbles, with experimental data present in the literature. Most of these studies show a reasonable agreement between experiments and simulations regarding the bed dynamics (e.g. pressure signals) and bubble behavior, but they are restricted to $2 \mathrm{D}$ bubbling beds.

There are also studies on the bed dynamics and bubble characteristics that use two dimensional numerical domains to represent three dimensional ( $3 \mathrm{D}$ ) systems. That is the case of McKeen and Pugsley (2003), who used a 2 D two fluid CFD model to simulate a $3 \mathrm{D}$ freely bubbling bed of FCC particles. Their simulations results of time averaged radial voidage profiles, radially averaged solids volume fraction and bed expansion were compared to experimental data, extracted from electrical capaci tance tomography. Johansson et al. (2006) simulated a fluidized bed operating in the slugging regime. As a validation, they evaluated their results with the power spectral density distribu tion of the fluctuating pressure signal and with local bubble parameters obtained experimentally with capacitance probes signals provided by other authors. Ahuja and Patwardhan (2008) compared their simulation and experimental results of solids hold up in a bubbling fluidized bed and studied the effect of geometrical parameters such as internals and gas distributor configuration. In that study, experiments were carried out in a cylindrical column using gamma ray tomography, whereas the simulation of the bed was done in a $2 \mathrm{D}$ domain. A cylindrical laboratory reactor was modeled in two dimensions by Hulme et al. (2005). These authors conducted a parametric study to determine the effect of time step, differencing scheme, closing equations and frictional stress in the simulation. The bubble properties, such as the average bubble diameter, were determined from maps of solids fraction using different cut off voidages $(0.3$, 0.2 and 0.15 ), which showed that the definition of the cut off is important to determine the bubble boundary.

However, it is not clear if two dimensional simulations can always be used as a reliable tool to reproduce the bed dynamics and bubble behavior in $3 \mathrm{D}$ beds. At this regard, the limit of the use of $2 \mathrm{D}$ models to study particular implementations of $3 \mathrm{D}$ systems has been subject of analysis in several works. Peirano et al. (2001) studied, in a statistically stationary bubbling flui dized bed of rectangular section (one lateral length much shorter than the other), the differences between $2 \mathrm{D}$ and $3 \mathrm{D}$ simulations by comparing their numerical simulations to experimental data concerning the power spectra of pressure fluctuations, the bed height and the probability distribution function of the particle volume fraction. They concluded that there may be significant differences between $2 \mathrm{D}$ and $3 \mathrm{D}$ simulations, pointing out that 2 D simulations can only be used for sensitivity analysis, and that quantitative validation must be done in $3 \mathrm{D}$. Moreover, they found that only $3 \mathrm{D}$ simulations can predict the bed height and the pressure spectra of the bed, because of the natural three dimensionality of the flow. More recently, Xie et al. (2008) presented the range of validity of $2 \mathrm{D}$ simulations to approximate both cylindrical and rectangular fluidized beds. The comparison with full $3 \mathrm{D}$ simulations was focused on the bed height, and the time averaged values of void fraction and velocity of gas and solids at different heights in bubbling, slugging and turbulent regimes, showing that discrepancies can be significant when the gas superficial velocity is sufficiently high (i.e. $U \geq 1.85 U_{m f}$ ) to produce bubbles of final size comparable to the bed width.

Despite the above commented differences between $2 \mathrm{D}$ and $3 \mathrm{D}$ simulations, two fluid $3 \mathrm{D}$ simulations are comparatively scarce in the literature, probably because of their computational cost. Peirano et al. (2002) studied the influence of the air supply system in a freely bubbling fluidized bed of rectangular section. They simultaneously used pressure and optical probes at the same location and capacitance probes. Nevertheless, owing to the high noise level present in their optical signals, only the results from the capacitance probes were used for comparison with a two fluid simulation. In particular, results from the numerical simulation were validated against measurements of the bed height, the spatial distribution of solids and the pressure spectra. Peirano et al. (2002) found some significant differences between their numerical predictions and these measurements. According to their results, the probability density function of particle volume fraction leads to a peak value shifted towards a volume 
fraction equal to 0.5 for the experiments and 0.64 for the computa tion, and the pressure spectra obtained from simulations and experiments were markedly different depending on the bubbling mode, i.e. multiple or single bubbling modes, the first being well predicted by the simulation. As Peirano et al. (2002) pointed out, the small pressure drop in the distributor needed in the single bubbling mode would require the simulation of the entire air supply system (and not only the plenum and the distributor) as it is strongly coupled to the bed dynamics. They also concluded that a more rigorous estimation of the bed characteristics, such as mean dia meter and frequency of the bubbles, is necessary to analyze the level of similarity between simulations and experiments. Simulations of the bubbling fluidization of a Geldart group B material in a 2 D and 3 D rectangular bed were done by Cammarata et al. (2003), reporting results on bed expansion, bubble size and bubble hold up. They compared the bubble size with predictions given by the Darton's equation, but the methodology used for the bubble volume calculation at a given height in $3 \mathrm{D}$ simulations was not detailed. Wang et al. (2010) presented a modification on the drag correlations to simulate industrial scale bubbling fluidized beds of Geldart B and D particles with a coarse computational grid. They compared their simulation results for the axial distribution of solids volume fraction and the average bed voidage with experimental measurements found in the literature.

These works reveal that two fluid models can be used as an efficient tool for the understanding of the hydrodynamics of fluidized beds. Nevertheless, it is also evident that there are few simulation studies where both bubble properties and dynamic characteristics of the bed (pressure) are simultaneously provided. Furthermore, this kind of combined information is lacking in the literature for $3 \mathrm{D}$ simulations, although it seems crucial to simulate the bed in a three dimensional domain in order to preserve all the degrees of freedom in the bubble and particle phase motion present in bed columns. For example, a $2 \mathrm{D}$ simulation based on the axisymmetry hypothesis could neither satisfactorily reproduce the alternating bubble paths in azimuth direction nor the coalescence of bubbles coming from two positions at the same height and radius in a cylindrical bed. Therefore, as remarked in Peirano et al. (2001), 3 D models should be used to reproduce realistically the bed dynamics.

The present study performs a two fluid (Eulerian Eulerian) $3 \mathrm{D}$ simulation of a cylindrical bed containing Geldard B particles flui dized in bubbling regime. The results from this $3 \mathrm{D}$ simulation are compared with the ones obtained from pressure and optical probe measurements for a real bed of similar dimensions and operative conditions. The ultimate objective of the present comparison is the characterization of both the dynamics of the bubbling regime and the bubble behavior in the bed. Sobrino et al. (2009a, 2009b) experimentally studied the bubble characteristics in a bubbling fluidized bed, where the bubbles were detected and their sizes were measured using optical fiber probes and the much more extensively employed pressure probes. What was experimentally obtained were the chord lengths of the bubbles, and by means of the maximum entropy method (MEM), bubble size probability density functions were calculated. In the present study, as a novelty, the same MEM is used to compare the experimental and simulation results. By this way, a comprehensive analysis of both signals, simulated and measured, is done, allowing a critical description and interpretation of the bubble behavior within a large fraction of the bed volume.

\section{Experimental set-up}

Experiments were carried out in a bubbling fluidized bed of $0.193 \mathrm{~m}$ internal diameter and $0.8 \mathrm{~m}$ height. The superficial gas velocity for the experiments was $U_{g}=0.57 \mathrm{~m} / \mathrm{s}$ and the minimum fluidization velocity of the bed was $U_{m f}=0.4 \mathrm{~m} / \mathrm{s}$. The bed was filled with Geldart B silica sand particles with a density of $2632.5 \mathrm{~kg} / \mathrm{m}^{3}$ and particle size distribution of mean diameter $540 \mu \mathrm{m}$ and standard deviation $75 \mu \mathrm{m}$. The settled bed height was $0.22 \mathrm{~m}$. The column had a perforated plate to distribute the air through 90 holes of $2 \mathrm{~mm}$ diameter laid out in a hexagonal pitch of $15 \mathrm{~mm}$. The total open area ratio of the distributor was $1 \%$. The distributor to bed pressure drop ratio was 0.5 at the minimum fluidization velocity and 0.9 at the nominal gas velocity in the experiment, $U_{g}$, ensuring a stable and homogeneous bubble generation. Pressure and optical probes were introduced in the bed in order to detect the bubble passage along the axial and radial directions. A sketch of the experimental set up can be seen in Fig. 1.

Optical probe and pressure measurements were performed at different positions inside the bed, with a duration of 10 minutes and a sampling frequency of $500 \mathrm{~Hz}$.

The optical probes developed for this study are based on backscattering principle (Liu et al., 2003). The optical fiber probes (OFP) were made of two standard step index plastic optical fibers (emitter and receiver fibers) embedded in a metallic coil of $3 \mathrm{~mm}$ external diameter. The fibers had a diameter of $1 \mathrm{~mm}$ with $0.22 \mathrm{~dB} / \mathrm{m}$ attenuation and $0.47 \mathrm{NA}$. The propagation delay con stant of the fiber was $5 \mathrm{~ns} / \mathrm{m}$ and the whole probe had a length of about $1 \mathrm{~m}$. The emitter fiber was illuminated by a $650 \mathrm{~nm}$ laser diode with a maximum power of $10 \mathrm{~mW}$ (Roithner s6510mg). A phototransistor was used at the reception as part of a transimpe dance amplifier, giving an output voltage proportional to the output optical power. The laser diode and the phototransistor were encapsulated in ST connectors. A 50/50 passive splitter was used for splitting the optical power into the emitter fibers of two probes. In order to measure the pierced length and velocity of the ascending bubbles, these two probes ( $\mathrm{op}_{1}$ and $\mathrm{op}_{2}$ in Fig. 1 ) were placed one above the other $1 \mathrm{~cm}$ apart.

Gage pressure measurements were carried out using piezo resistive differential pressure transducers Omega PX291 (0 5 in $\mathrm{H}_{2} \mathrm{O}$ ) with a $1 \% \mathrm{FS}$ accuracy. The high pressure port was connected to a probe immersed in the bed and the low pressure port was exposed to the atmosphere.

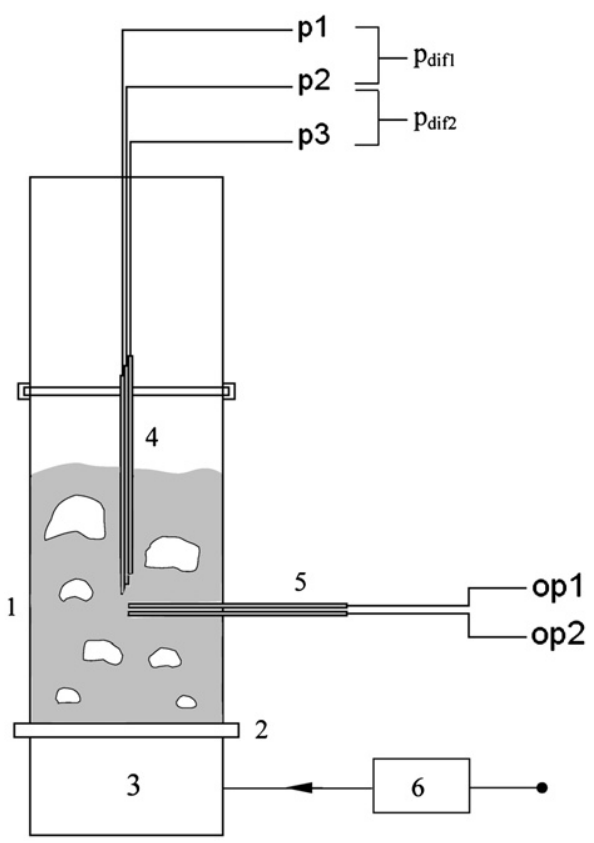

Fig. 1. Experimental set-up: (1) fluidized bed column, (2) distributor plate, (3) plenum chamber, (4) pressure probes, (5) optical probes and (6) air flow meter. 
Three pressure probes $\left(p_{1}, p_{2}\right.$ and $p_{3}$ in Fig. 1$)$ of $4 \mathrm{~mm}$ external diameter were placed inside the bed opposed to the flow direc tion and separated an axial distance of $1 \mathrm{~cm}$ from each other. As shown in Fig. 1, two differential pressure signals can be recorded employing this configuration $p_{\text {dif } 1}=p_{1} \quad p_{2}$, and a second one $p_{\text {dif } 2}=p_{2} \quad p_{3}$.

\section{Two-fluid CFD model}

A CFD simulation of the fluidized bed was performed using an Eulerian description of both the gas and particle phases by means of a two fluid model (van Wachem and Almstedt, 2003). In particular, this model makes use of the following equations for the conservation of mass and momentum in both the gas phase $(g)$ and particulate or solids phase $(p)$, the last phase treated as a fluid with effective transport properties (Gidaspow, 1994):

$\frac{\partial}{\partial t}\left(\alpha_{g} \rho_{g}\right)+\nabla\left(\alpha_{g} \rho_{g} \mathbf{v}_{g}\right)=0$

$\frac{\partial}{\partial t}\left(\alpha_{p} \rho_{p}\right)+\nabla\left(\alpha_{p} \rho_{p} \mathbf{v}_{p}\right)=0$

$\frac{\partial}{\partial t}\left(\alpha_{g} \rho_{g} \mathbf{v}_{g}\right)+\nabla\left(\alpha_{g} \rho_{g} \mathbf{v}_{g} \mathbf{v}_{g}\right)=\alpha_{g} \nabla p+\nabla\left(\alpha_{g} \tau_{g}\right) \quad K_{g p}\left(\mathbf{v}_{g} \quad \mathbf{v}_{p}\right)+\alpha_{g} \rho_{g} \mathbf{g}$

$\frac{\partial}{\partial t}\left(\alpha_{p} \rho_{p} \mathbf{v}_{p}\right)+\nabla\left(\alpha_{p} \rho_{p} \mathbf{v}_{p} \mathbf{v}_{p}\right)=\alpha_{p} \nabla p \quad \nabla p_{p}+\nabla\left(\alpha_{p} \tau_{p}\right)+K_{g p}\left(\mathbf{v}_{g} \quad \mathbf{v}_{p}\right)+\alpha_{p} \rho_{p} \mathbf{g}$

where $\alpha_{g}+\alpha_{p}=1$ and $\tau_{i}=\mu_{i}\left(\nabla \mathbf{v}_{i}+\nabla \mathbf{v}_{i}^{T}\right)+\left(\lambda_{i} \quad(2 / 3) \mu_{i}\right)\left(\nabla \mathbf{v}_{i}\right) \mathbf{I}$ for any phase " $i$ ". The viscosity for the particulate phase in $\tau_{p}$ is composed of the collisional, kinematic and frictional viscosity $\mu_{p}=\mu_{p, c o l}+\mu_{p, k i n}+\mu_{p, f}$.

The mass and momentum equations are solved together with the differential equation for the transport of granular temperature $\Theta$ (Gidaspow, 1994):

$$
\begin{aligned}
& \frac{3}{2}\left[\frac{\partial}{\partial t}\left(\rho_{p} \alpha_{p} \Theta\right)+\nabla\left(\rho_{p} \alpha_{p} \Theta \mathbf{v}_{\mathbf{p}}\right)\right]=\left(p_{p} \mathbf{I}+\tau_{p}\right): \nabla \mathbf{v}_{\mathbf{p}} \\
& +\nabla\left(k_{\Theta} \nabla \Theta\right) \quad \gamma_{\Theta} 3 K_{g s} \Theta
\end{aligned}
$$

which is based on the kinetic theory of granular flows and provides the level of random fluctuation of particle velocity due to collisions. Notice that the granular temperature is required for the closure expressions of the drag coefficient $K_{g p}$, the solid viscosities $\mu_{p}$ and $\lambda_{p}$, and the effective particle pressure $p_{p}$. The diffusion coefficient of granular temperature $k_{\Theta}$ and the collision dissipation energy $\gamma_{\Theta}$ are also functions of $\Theta$. For the drag coefficient, the closure equation of Gidaspow et al. (1992) has been chosen due to its robustness at the beginning of the simulation sequence, which commences with air entering the distributor with the bed at rest and particle volume fraction $\alpha_{p \text {,max }}$. Table 1 summarizes these and other closure models selected for the present study.

The commercial CFD software Fluent 6.3 (Fluent Inc., 2006) was used for the solution of the system of Eqs. (1) (5) in a $3 \mathrm{D}$ domain comprising all the interior volume of the cylinder where the fluidized bed is allocated. The dimensions of the cylinder are equal to the ones of the experimental rig. The domain was discretized with a boundary fitted mesh of 28,800 hexahedral cells and 30,805 nodes. The code discretizes each of the equations with an implicit finite volume technique applied in any of the cells of the domain (Patankar, 1980). Due to the great complexity and number of equations involved, a larger amount of cells would lead to an inadmissible time of computation. However, to improve the spatial and temporal resolution of the solution, second order discretization in space and time was selected. After a sensitivity analysis of the solution, the chosen time step was
Table 1

Summary of closure models.

A. Coefficient of drag between gas and particles (Gidaspow et al., 1992)

$K_{g p} \quad \frac{3}{4} C_{D} \frac{\alpha_{p} \alpha_{g} \rho_{g}\left|\mathbf{v}_{p}-\mathbf{v}_{g}\right|}{d_{p}} \alpha_{g}{ }^{265}$ for $\alpha_{g}>0.8$

$K_{g p} \quad 150 \frac{\alpha_{p}^{2} \mu_{g}}{\alpha_{g} d_{p}^{2}}+1.75 \frac{\alpha_{g} \rho_{g}\left|\mathbf{v}_{p}-\mathbf{v}_{g}\right|}{d_{p}}$ for $\alpha_{g} \leq 0.8$

where the drag coefficient is defined as

$C_{D} \frac{24}{\alpha_{g} \operatorname{Re}_{p}}\left[1+0.15\left(\alpha_{g} \operatorname{Re}_{p}\right)^{0687}\right]$

with

$\operatorname{Re}_{p} \frac{\rho_{g} d_{p}\left|\mathbf{v}_{p}-\mathbf{v}_{g}\right|}{\mu_{g}}$

B. Solids pressure (Lun et al., 1984)

$p_{p} \quad \alpha_{p} \rho_{p} \Theta+2 \rho_{p}\left(1+e_{p p}\right) \alpha_{p}^{2} g_{0, p p} \Theta$

where the radial distribution function is

$g_{0, p p}\left[1-\left(\frac{\alpha_{p}}{\alpha_{p, \max }}\right)^{1 / 3}\right]^{1}$

C. Solids stress tensor

$\tau_{p} \quad \alpha_{p} \mu_{p}\left(\nabla \mathbf{v}_{p}+\nabla \mathbf{v}_{p}^{\mathrm{T}}\right)+\alpha_{p}\left(\lambda_{p}-\frac{2}{3} \mu_{p}\right) \nabla \mathbf{v}_{p} \mathbf{I}$

where solid bulk viscosity is (Lun et al., 1984)

$\lambda_{p} \quad \frac{4}{3} \alpha_{p} \rho_{p} d_{p} g_{0, p p}\left(1+e_{p p}\right)\left(\frac{\Theta}{\pi}\right)^{1 / 2}$

and solid shear viscosity is

$\mu_{p} \quad \mu_{p, \text { col }}+\mu_{p, \text { fr }}+\mu_{p, k i n}$

which is composed of a kinetic viscosity (Gidaspow et al., 1992):

$\mu_{p, k i n} \frac{10 \rho_{p} d_{p} \sqrt{ } \Theta \pi}{96 \alpha_{p}\left(1+e_{p p}\right) g_{0 p p}}\left[1+\frac{4}{5} g_{0 p p} \alpha_{p}\left(1+e_{p p}\right)\right]^{2}$

a collisional viscosity (Gidaspow et al., 1992):

$\mu_{p, \text { col }} \quad \frac{4}{5} \alpha_{p} \rho_{p} d_{p} g_{0, p p}\left(1+e_{p p}\right)\left(\frac{\Theta}{\pi}\right)^{1 / 2}$

and a frictional viscosity (Schaeffer, 1987):

$\mu_{p, f r} \frac{p_{p} \sin \phi}{2 \sqrt{ } I_{2 D}}$

where $\phi$ is the angle of internal friction, and $I_{2 D}$ is the second invariant of the deviatory stress tensor.

D. Diffusion coefficient of granular temperature (Gidaspow et al., 1992)

$k_{\Theta} \frac{150 \rho_{p} d_{p} \sqrt{ } \Theta \pi}{384\left(1+e_{p p}\right) g_{0 p p}}\left[1+\frac{6}{5} \alpha_{p} g_{0 p p}\left(1+e_{p p}\right)\right]^{2}$

$+2 \rho_{p} d_{p} \alpha_{p}^{2} g_{0 p p}\left(1+e_{p p}\right) \sqrt{\frac{\Theta}{\pi}}$

E. Collisional dissipation of energy (Lun et al., 1984)

$\gamma_{\Theta} \frac{12\left(1-e_{p p}^{2}\right) g_{0, p p}}{d_{p} \sqrt{ } \pi \rho_{p} \alpha_{p}^{2} \Theta^{3 / 2}}$

equal to $2.5 \mathrm{e} 4 \mathrm{~s}$ with 40 iterations per time step together with an algebraic multigrid methodology for the solution of the implicit system equations (Hutchinson and Raithby, 1986). An effective value of the coefficient of restitution $e_{p p}=0.9$ has been chosen to take into account not only the dissipation of kinetic energy due to inelastic deformation of particles but also due to frictional losses (Goldschmidt et al. 2001). For the simulation, particles are assumed spherical and monodispersed with a size equal to the mean diameter of the particles in the experiment. Other parameters of the simulation are shown in Table 2 .

Regarding the boundary conditions of the computational domain, shown in Fig. 2, the distributor was modeled as a porous 
Table 2

Main parameters selected for the simulation.

\begin{tabular}{lclc}
\hline Parameter & Value & Parameter & Value \\
\hline$R(\mathrm{~m})$ & 0.0965 & $\alpha_{p, \max }($ dimensionless $)$ & 0.555 \\
$H(\mathrm{~m})$ & 1 & $h_{0}(\mathrm{~m})$ & 0.22 \\
$\rho_{g}\left(\mathrm{~kg} / \mathrm{m}^{3}\right)$ & 1.225 & $U_{g}(\mathrm{~m} / \mathrm{s})$ & 0.57 \\
$\mu_{g}(\mathrm{~Pa} \mathrm{~s})$ & $1.789 \mathrm{e}-5$ & $g\left(\mathrm{~m} / \mathrm{s}^{2}\right)$ & 9.81 \\
$d_{p}(\mu \mathrm{m})$ & 540 & $\theta_{\text {fric }}(\mathrm{deg})$. & 30 \\
$\rho_{p}\left(\mathrm{~kg} / \mathrm{m}^{3}\right)$ & 2632.5 & $\Delta t(\mathrm{~s})$ & $2.5 \mathrm{e}-4$ \\
$e_{p p}($ dimensionless $)$ & 0.9 & $N_{i}$ & 40 \\
\hline
\end{tabular}

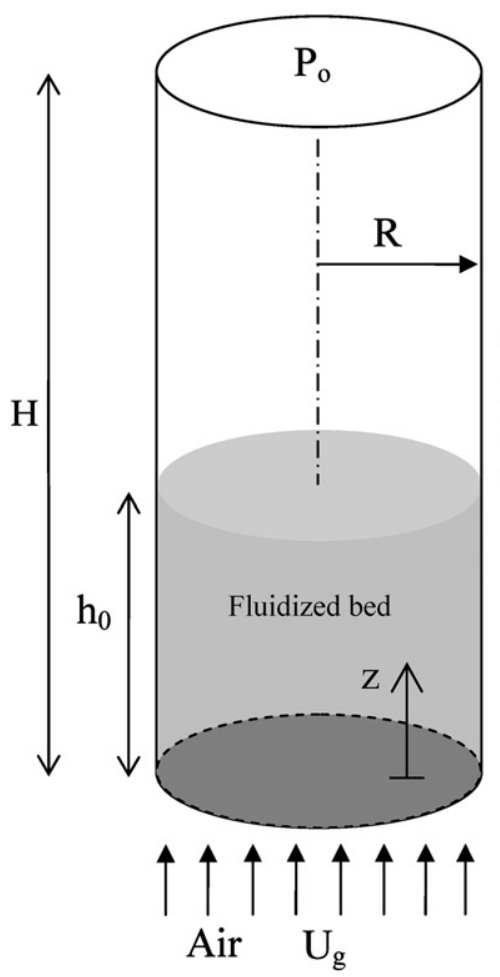

Top:

Pressure outlet

$1 \mathrm{~atm}$

Walls:

Non-slip condition for air Zero particle-wall shear stresses

Distributor:

homogenous velocity inlet

Fig. 2. Computational domain and boundary conditions used in the simulation.

plate placed at the base of the cylinder through which air is uniformly (in time and space) injected into the fluidized bed. Particles are not allowed to cross the distributor. The top of the cylinder is assumed to have a constant static pressure of one atmosphere, since it is open to the exterior air. A no slip condition for the gas flow was imposed at the lateral wall of the cylinder. For the dense phase flow, this condition is relaxed to non penetration with negligible shear stresses at the lateral walls, since, in this kind of bed configuration, particles are well fluidized and do not remain attached to the wall. Hence, in the present cylindrical bed, the effects of the particle wall shear stresses on the interior of the bed volume are negligible. Besides, an imposi tion of other boundary conditions for the particle phase such as no slip or partial slip conditions would require, to be properly imposed, a very fine mesh near the lateral walls in order to estimate the gradients of particle phase velocity needed in these conditions.

A series of virtual measurement points were defined within the volume of the bed with the aim of sampling pressure and particle volume fraction in the exact positions and conditions of the pressure and optical probes, respectively, that are employed in the experiments. The simulation covered a long period of real time in fully bubbling regime after passing the fluidization startup from the initial conditions. In particular, the pressure and particle volume fraction signals taken from the virtual measurement points were acquired for an uninterrupted period of $60 \mathrm{~s}$ of real time, ensuring with this that the results were statistically representative.

\section{Signal processing}

\subsection{Bubble parameters determination from pressure and optical/ particle volume fraction signals}

In order to perform an unbiased comparison between the real bed and the simulated one, their signals should be treated with equal techniques. Bubble velocity and pierced length (i.e. bubble chord) can be calculated from the simulated differential pressure signals ( $p_{\text {dif } 1}$ and $p_{\text {dif } 2}$ in Fig. 1 ), assuming that the pressure field around a rising bubble in a fluidized bed is well represented by the classical Davidson model (Davidson and Harrison, 1963). The bubble velocity is calculated dividing the vertical distance between the pressure probes $(1 \mathrm{~cm})$ by the time that the bubble takes to travel from the lower to the upper probe. This time can be approximated to $\Delta t_{S}$, which is the mean of offset times between the two differential pressure signals (see example in Fig. 3):

$\Delta t_{S}=\frac{\Delta t_{i}+\Delta t_{0}}{2}$

where $\Delta t_{i}=t_{i 2} \quad t_{i 1}$ and $\Delta t_{o}=t_{o 2} \quad t_{o 1}$, with $t_{i}$ and $t_{o}$ being the time instants at which the differential pressure crosses the detection threshold. This procedure is also used by Liu et al. (2010) in the framework of optical probe signals. In the present work, the threshold for the differential pressure signals has been selected to be equal to the mean value of the whole pressure signal. The bubble pierced length is then calculated multiplying the bubble rise velocity by the bubble passage time. The bubble passage time is the period during which a differential pressure signal falls below its average (Ramayya et al. 1996). Therefore, $\Delta t_{S}$ in Eq. (6) can be also inter preted as the difference between centers of bubble passage times in the two probes, $\Delta t_{S}=t_{c 2} \quad t_{c 1}$, with $t_{c}=\left(t_{i}+t_{o}\right) / 2$ as shown in Fig. 3. However, due to the fact that the differential pressure signal may be also perturbed by bubbles not crossing the measurement points, bubbles rising with a horizontal eccentricity greater than the bubble radius, i.e. bubbles for which the differential pressure record falls less than half of its average value, have been rejected (Dent et al., 1989). This transforms the effective threshold for the detection of

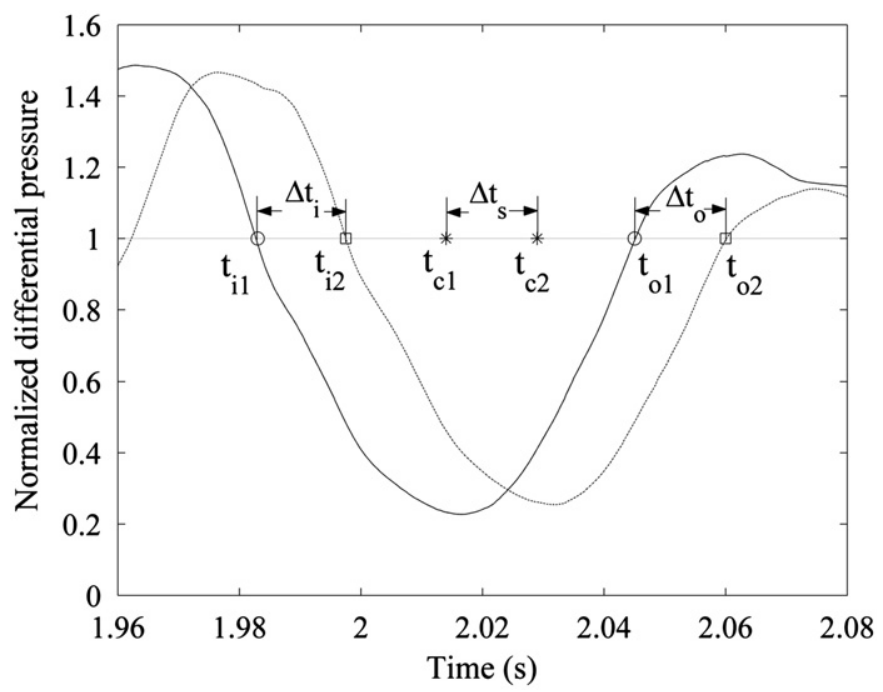

Fig. 3. Estimation of the bubble passage time using two differential pressure signals from 3 vertically aligned probes. 
bubbles to a conservative figure equal to half the value of the time average differential pressure.

For the detection of bubbles using the experimental optical probe, when the signal falls below a threshold voltage, it is considered that a bubble passage is occurring. However, owing to the high stochasticity of the backscattered light in the bed, the threshold for optical signals should be determined by plotting the probability density function (PDF) of the voltage signal from the probe. The PDF exhibits a peak corresponding to the emulsion phase and a tail at lower voltages corresponding to gas bubbles (Schweitzer et al., 2001). The bubble detection threshold has been defined as the voltage where the histogram tail begins, i.e. where the slope of the histogram becomes zero or nearly zero (Sobrino et al., 2009a). Once a bubble has been detected in the optical signals, the bubble passage time is estimated as the local period of time in which the optical signal falls below the time average value of the entire signal, as in the case of the differential pressure. Observe that the time average value of the optical signal is a robust parameter relatively independent of the random noise that affects the experimental signal. Following the work of Liu et al. (2010), the rise velocity of bubbles is calculated dividing the vertical separation of the lower and the upper optical probes (op 1 and $o p_{2}$ in Fig. 1 ) by the time taken by the bubble to travel from one probe to the other, $\Delta t_{s}$, calculated as explained pre viously for the pressure signals.

Optical probe signals and simulated particle fraction signals are both related to the distribution of the emulsion and the gaseous phases within the bed. Nevertheless, the detection of a bubble in the simulated signal is not affected by measurement noise. In the present work, a particle volume fraction equal to the time average volume fraction, in each measurement point, has been directly used as an unrestrictive threshold for the bubble detection. The bubble pierced length sensitivity to this threshold is shown in Section 5.3.1. Once the bubble is detected, a procedure similar to the one described for optical signals has been used to calculate the bubble velocity and pierced length.

For both the simulation and the experimental signals, the following criteria are applied to reject erroneous bubble size measurements: pierced length measurements smaller than the separation between probes are considered potentially inaccurate, and pierced lengths larger than the bed radius are treated as outliers (e.g. coalescent or erroneously detected bubbles) as they will lead to bubble diameters of the order of the column diameter, neither observed in the experiment nor in the simulation. By this way, the contamination of results with erroneous estimations of the bubble pierced length and velocity is mitigated. Note that the uncertainties of the velocity and pierced length measurements can be calculated by propagating the uncertainties of the contributing parameters (Sobrino et al., 2009a) and they reach a maximum value of $0.1 \mathrm{~m} / \mathrm{s}$ and $8 \mathrm{~mm}$ respectively for the experimental data and $0.02 \mathrm{~m} / \mathrm{s}$ and $1 \mathrm{~mm}$ respectively for the simulated data.

\subsection{Determination of the probability density function of the bubble pierced length and diameter}

Using the bubble pierced length samples obtained from experi mental and simulation signals, the probability density function of the bubble size was estimated applying the maximum entropy method (Santana et al., 2006). This method is employed to obtain the probability density function of a variable provided the distribution is subjected to a certain number of constrains that are known, such as the geometric moments of the variable distribution. For the bubble pierced length this procedure is straightforward, as the geometric moments of the pierced length distribution can be directly estimated using the sampled pierced lengths extracted from the measured or the simulated signals as explained in the previous section. The raw moments of the bubble diameter distribution need to be estimated from the bubble pierced lengths deducing the equations that relate both magnitudes. The estimation of this relation entails some assumptions: bubbles are assumed to rise randomly distributed in a horizontal surface of radius equal to the bubble radius surrounding the probe, in the sense that the probability of the bubble to be pierced at any point of its surface is the same (Werther 1974); the ascending velocity of the bubble is considered vertical, and the bubble geometry has been modeled as a truncated spheroid, which is the most general shape adopted by the bubbles in a fluidized bed. A detailed explanation of the equations applied to estimate the bubble size distribution using the maximum entropy method can be found in Sobrino et al. (2009a).

\section{Results and discussion}

\subsection{General dynamics}

Pressure signals, taken from the experiments and the simulation of the fluidized bed for a representative period of time, are shown in Fig. 4. In the same figure, the resulting optical probe measurements and the simulated particle volume fraction are also plotted. The values depicted correspond to the same period of time (i.e. simulta neous acquisition of differential pressure and optical or particle fraction signals). In the case of the differential pressure, Fig. 4a and c, it is the difference between pressures at heights $z_{1}=12 \mathrm{~cm}$ and $z_{2}=13 \mathrm{~cm}$, and mid radius $r / R=0.5$. The optical probe signal and the simulated particle fraction are plotted in Fig. $4 \mathrm{~b}$ and $\mathrm{d}$ for a point at $z=12 \mathrm{~cm}$ and $r / R=0.5$ in the bed.

Experimental and simulated signals in Fig. 4 are the raw acquired data. Neither hardware nor digital filtering has been performed in order to compare the data free of the effect of any signal conditioning. High frequency oscillation of the optical signal in Fig. 4b is due to the stochastic character of the light backscattering at high particle concentration (i.e. outside bub bles). Notice that, although filtering of the optical signal may improve its appearance, it is an operation that can also destroy valuable information regarding the signal spectrum and bubble size parameters. It is evident from Fig. 4 that the real bed and the simulated one yield signals of very similar character in both the differential pressure and the optical signal/particle fraction cases. The fluctuation amplitude of the differential pressure measured by the probes compares also well with the simulation results.

Fig. 5 shows a series of snapshots of the instantaneous void distribution (i.e. bubbles) in the $3 \mathrm{D}$ bed obtained from the two fluid model simulation. The aim of Fig. 5 is to provide a physical interpretation of the signal oscillations depicted in Fig. 4. The snapshots of Fig. 5 correspond to four consecutive instants of time, named as 1, 2, 3 and 4, which are indicated in Fig. 4c and d. The bubbles shown in Fig. 5 have been extracted from iso surfaces of void fraction equal to 0.7, that is, a threshold in particle volume fraction $\alpha_{p, t h}=0.3$. The particle volume fraction shown in the vertical planes of Fig. 5 indicates that the volume fraction gradients are very concentrated near the bubbles surface and in their wake, the rest of the volume outside bubbles having a homogenous particle volume fraction close to $\alpha_{p, \max }$ of Table 2 . As Fig. 5 illustrates, a bubble laterally crosses the virtual measure ment points that are placed in the simulation domain at $r / R=0.5$ and $z$ equal to 12 and $13 \mathrm{~cm}$. This creates a perturbation in the simulated differential pressure signal (Fig. 4c), which resembles the theoretical difference pressure obtained with the classical two phase model around a rising bubble (Davidson and Harrison, 1963). In Fig. 5a the bubble is approaching the virtual measure ment probes, thus creating an increase of the differential pressure as Fig. 4c confirms. The differential pressure in Fig. 4c decays from 
a

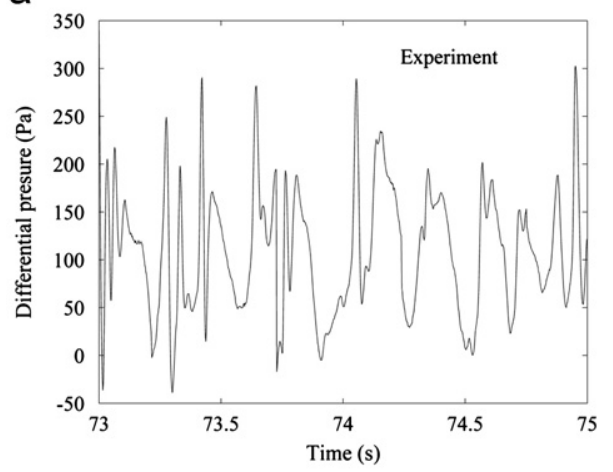

C

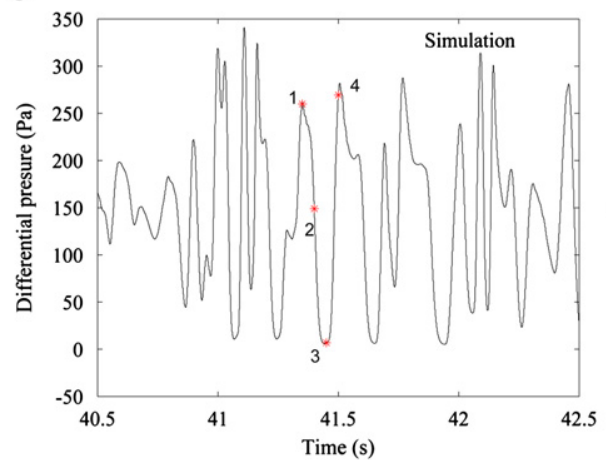

b

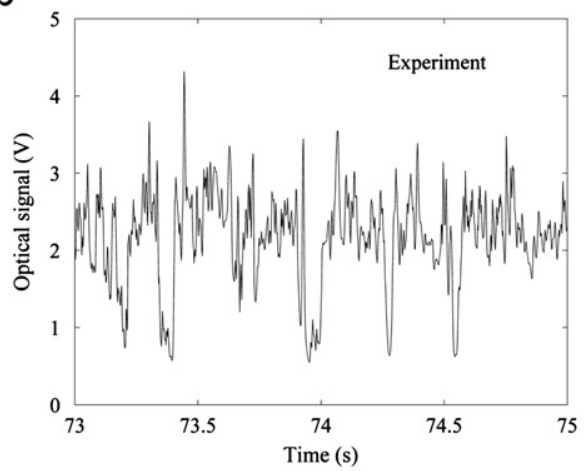

d

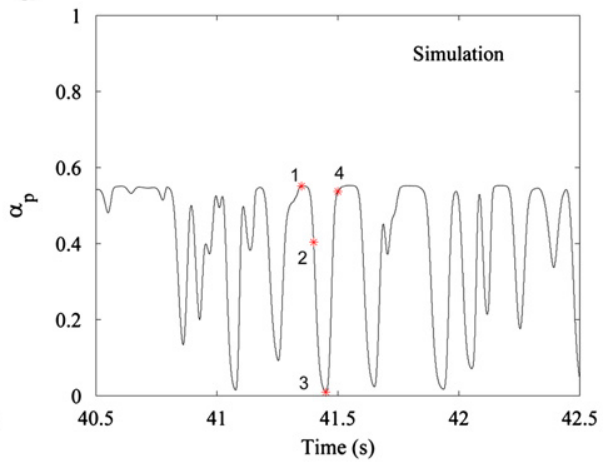

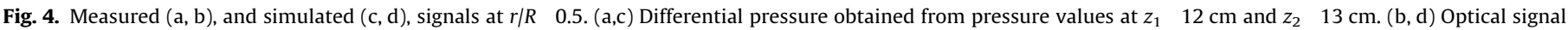
and particle fraction at $z_{1} 12 \mathrm{~cm}$.

point 1 to a minimum in point 3 since the bubble is crossing the virtual probes (Fig. 5b and c). Finally, the differential pressure is recovered and reaches a second maximum in Fig. 4c just after the passing of the bubble, as shown in Fig. $5 \mathrm{~d}$. The passing of a bubble is also clearly detected in Fig. 4d, in which there is a decrease in the simulated particle fraction in the time interval from point 1 to point 4 . It is to be noticed that the optical or the particle volume fraction signals are affected only when a bubble crosses the acquisition point. In contrast, as pressure propagates through the emulsion phase, pressure signals may be additionally perturbed by bubbles passing near the acquisition point but not necessarily crossing it. That explains why the experimental and simulated differential pressure signals, in Fig. 4a and c, have a greater number of major oscillations than optical and volume fraction signals, Fig. $4 \mathrm{~b}$ and d.

\subsection{Frequency analysis}

The power spectral densities (PSD) for the pressure, the pre ssure difference, and the particle volume fraction/optical signals have been calculated in this work averaging the sub spectral densities of data sections in order to reduce their variance (Johnsson et al., 2000). In the present work, an average of the spectra from 8 consecutive sections of the signal with $50 \%$ overlapping and a Hamming window to smooth the signal at the section endpoints have been selected. Table 3 contains the most relevant peak frequencies of the spectra presented in Figs. 6 and 7.

Examining firstly the PSD of the absolute pressure, solid lines in Fig. 6, it is evident that the simulation provides a spectrum whose pattern is similar to the one obtained from experiments. A frequency shift lower than $1 \mathrm{~Hz}$ seems to differentiate both cases, but the same dynamics of the bed, i.e. distribution of peak frequencies and width of the spectrum, are clearly reflected.
Notice that the dominant frequency of absolute pressure, $f_{d}$, is in both the simulation and the experiments placed close to the natural frequency of the bed given by Baskakov et al. (1986):

$f_{e}=\frac{1}{\pi} \sqrt{\frac{g}{h_{m f}}}$

which is $2.1 \mathrm{~Hz}$ in the case of the bed studied here. The independence of $f_{e}$ to the measurement point is shown in Fig. 6, where the simulation results for a point near the distributor, i.e. at $z=7 \mathrm{~cm}$ and $r / R=0$, evidence that $f_{d}$ is preserved regardless the distance to the distributor.

It is well known that absolute pressure reflects global informa tion of the bed, whereas the differential pressure measured in a short spatial interval is more indicative of the local behavior. As reported in Roy et al. (1990) and Bi (2007), there are significant differences in both spectra, with a shift to higher frequencies in the case of the double probe results (i.e. differential pressure). This frequency shift is corroborated by the power spectral densities of pressure and differential pressure shown in Fig. 6 in both the simulated (Fig. 6a) and the experimental (Fig. 6b) signals. In the simulated case (Fig. 6a) the peak around $3.4 \mathrm{~Hz}$ has disappeared, while in the experimental PSD of pressure difference (Fig. 6b), this low frequency peak remains, though now it is not the only principal frequency. Besides another intensity peak at higher frequencies appears (i.e. $f_{d i f 1}=6.3 \mathrm{~Hz}$ in Table 3), which is coincident with the high amplitude frequency that comes out in the simulation $\left(f_{d i f 1}=6.5 \mathrm{~Hz}\right)$. These intensity peaks of the differential pressure spectra can be attributed to the local passing of bubbles through the acquisition points as justified later on. In the experiments, signals are affected by measurement noise, and this fact could contribute to the high frequency components of the spectrum, which are enhanced after the subtraction of signals during the calculation of the differential 
a

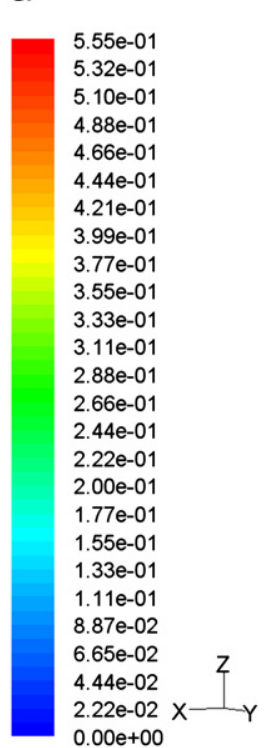

C

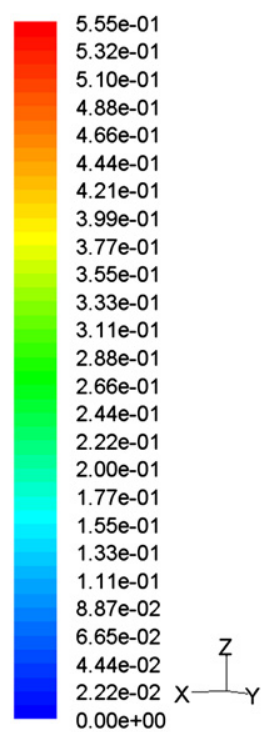

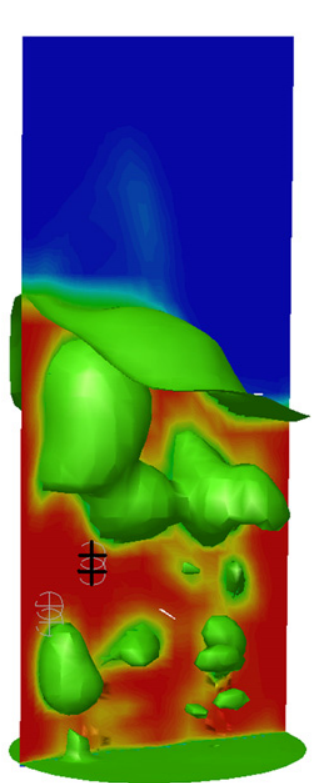

b

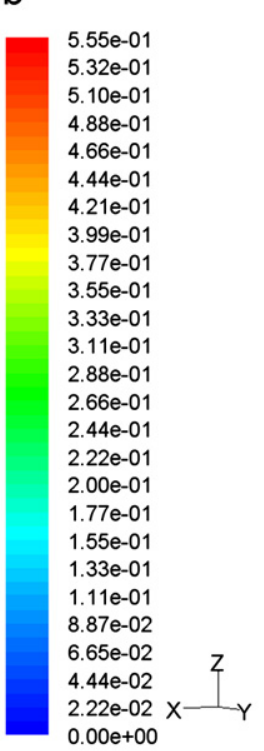

d

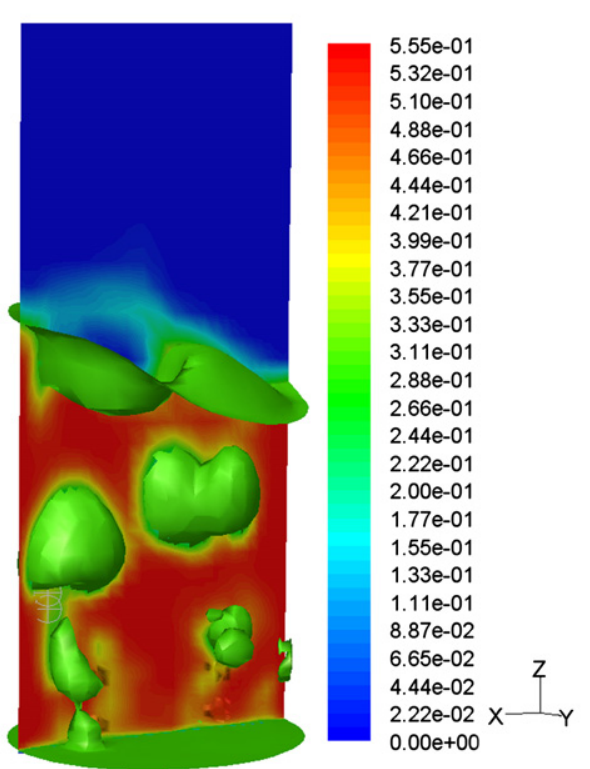

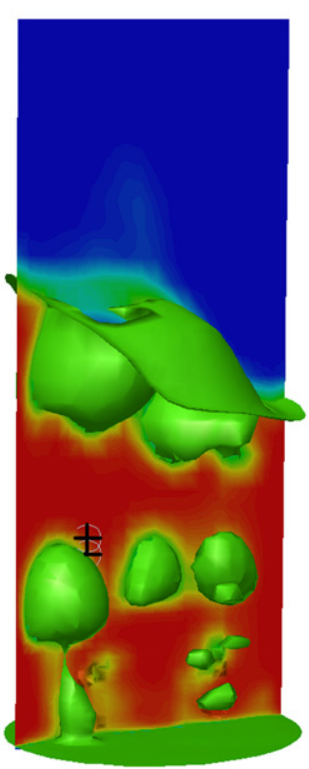

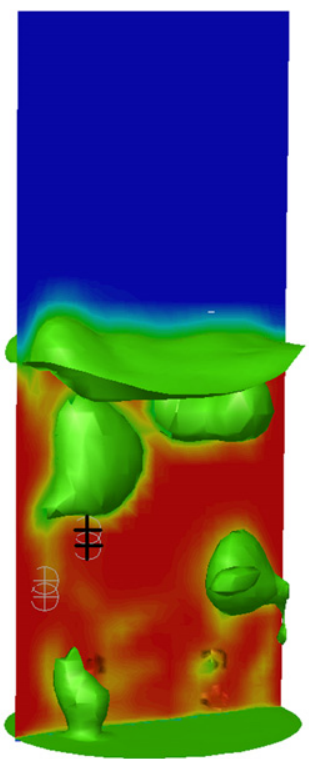

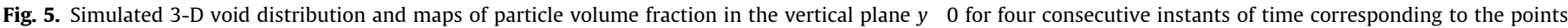
marked in Fig. 4: (a) point 1; (b) point 2; (c) point 3 and (d) point 4.

Table 3

Experimental and simulated characteristic frequencies of the PSD for absolute pressure, differential pressure and optical/volume fraction signals.

\begin{tabular}{lll}
\hline & Simulation & Experiments \\
\hline $\begin{array}{l}\text { Absolute pressure } \\
f_{d}(\mathrm{~Hz})\end{array}$ & 3.4 & 2.7 \\
Differential pressure & & \\
$f_{\text {dif1 }}(\mathrm{Hz})$ & 6.5 & 6.3 \\
$f_{\text {dif } 2}(\mathrm{~Hz})$ & 8.1 & 4.5 \\
Optical signal or particle volume fraction & \\
$f_{o p 1}(\mathrm{~Hz})$ & 6.4 & 6.8 \\
$f_{o p 2}(\mathrm{~Hz})$ & 8.0 & 4.3 \\
\hline
\end{tabular}

pressure. Nevertheless, the averaging of individual PSD to obtain the spectra presented in Fig. 6 reduces the effect of measurement noise at high frequencies.
Fig. 7 compares the resulting power spectral densities of the simulated solids fraction (Fig. 7a) and the experimental optical probe signals (Fig. 7b). Both signals are simultaneously taken from a point in the axis close to the points where pressure signals in Fig. 6 were measured. As bubbles not piercing the measurement points do not affect the volume fraction and the optical probe signals, the peak frequencies of these signals should coincide only with the bubble passing frequency at the measurement points. In the simulation results, it can be observed that the principal peak frequency of the particle fraction spectrum, i.e. $f_{o p 1}=6.4 \mathrm{~Hz}$ in Fig.7a, coincides with the high frequency peak of the differential pressure spectra, $f_{\text {dif } 1}=6.5 \mathrm{~Hz}$ in Fig. 6a. In the experiments, also a frequency peak, $f_{\text {op } 1}=6.8 \mathrm{~Hz}$, can be identified near the value $f_{\text {dif } 1}=6.3 \mathrm{~Hz}$ taken from the differential pressure spectrum. In this case the intensity peak of $f_{o p 1}$ is not as clear as in the simulation.

The second peak in the differential pressure spectrum from the simulation $\left(f_{d i f 2}=8.1 \mathrm{~Hz}\right.$ in Fig. 6a) has also a good matching in 
a

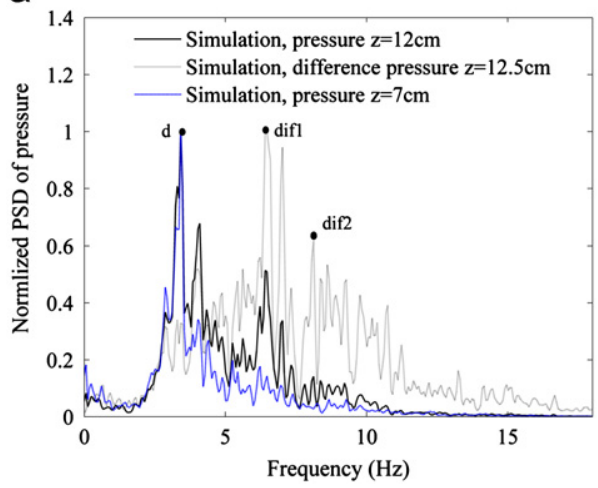

b

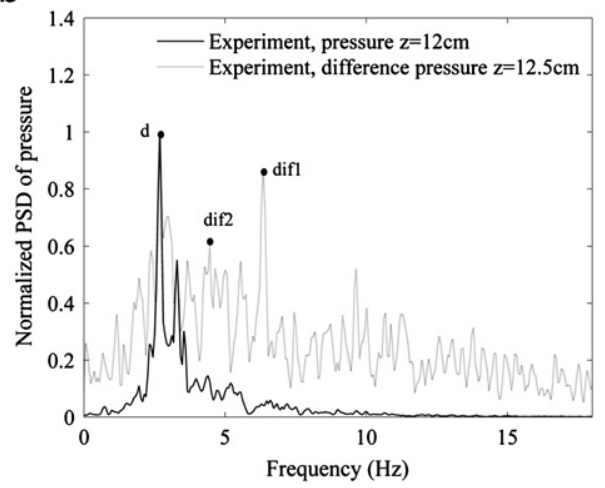

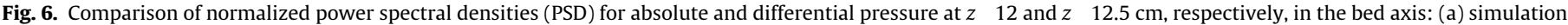
results and (b) experiment results.
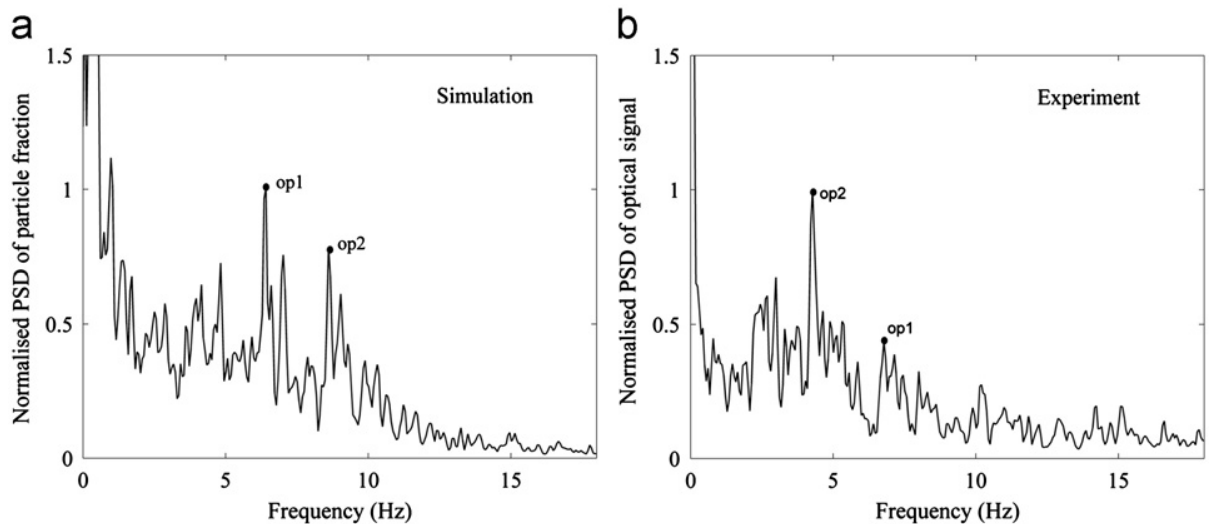

Fig. 7. Normalized power spectral densities (PSD) at $z \quad 10 \mathrm{~cm}$ in the bed axis for: (a) simulated particle volume fraction and (b) optical probe signal.

the particle fraction PSD $\left(f_{o p 2}=8.0 \mathrm{~Hz}\right.$ in Fig. 7a). In the experi mental case, there is also a frequency peak correspondence $\left(f_{\text {dif } 2}=4.5 \mathrm{~Hz}\right.$ in Fig. 6b and $f_{o p 2}=4.3 \mathrm{~Hz}$ in Fig. 7b). Differences in these last cases between experiments and simulation might be due, among other reasons, to the fact that the intensity level of these second peaks is more sensitive to the signals stochasticity and the measurement noise.

The resulting matching between $f_{\text {dif }}$ and $f_{\text {op }}$ corroborates the hypothesis that most of the higher frequency peaks of the differential pressure PSD are generated by the local dynamics of the bed, that is, by bubbles crossing the measurement points. Note that the intensity of the spectrum for both the simulation and experiments in Fig.7, increases when the frequency tends to null values, but this does not occur in Fig. 6. This is perhaps related to the shape of the optical and particle volume fraction signals, which may be roughly viewed as square wave signals alternating two levels (i.e. outside and inside bubble levels).

\subsection{Bubble characterization}

Optical signals were preferred in the present study to experi mentally determine the bubble parameters rather than experi mental pressure measurements because, near the distributor, the bed pressure probes were less reliable due to the small bubble size, as explained in Sobrino et al. (2009b). Nevertheless, even in the case of the small sized optical sensors, the presence of the probes in the real bed can interfere in the bubble dynamics. According to Rowe and Masson (1981), horizontally supported probes tend to decelerate bubbles and may promote splitting.

While experimental signals (Fig. 4a and b) present a charac teristic noise, intrinsic to the measurement chain, the simulated signals of pressure and particle fraction (Fig. 4c and d) are noise free and can both be more easily used for the bubble character ization. Nevertheless, the detection of the bubbles from the simulated differential pressure signals is restricted by the bubble detection threshold. This bubble detection threshold is more conservative than the one used for the particle volume fraction in the simulated signals, as explained in Section 4.1. Moreover, two consecutive differential pressure records are needed to determine the bubble properties, which means that bubbles have to cross a distance of $2 \mathrm{~cm}$ to be correctly measured, while for the case of particle fraction this distance is lower $(1 \mathrm{~cm})$. Therefore, a lower probability of detecting bubbles can be expected if differ ential pressure is considered.

\subsubsection{Sensitivity to the bubble detection threshold}

There is no consensus in the literature providing a unique value for the threshold of particle volume fraction which may define a bubble. In this section, the sensitivity of the simulation results to this threshold is presented. Cammarata et al., 2003 and Patil et al., 2005 considered a bubble as the coherent region within the fluidized bed where the solids volume fraction is lower than 0.15. van Wachem et al., 1998 and McKeen and Pugsley, 2003 assumed that the upper limit for the solids volume fraction within a bubble is 0.2. Peirano et al., 2001 distinguished between dense phase, where the particle volume fraction is close to the maximum packing, and dilute phase with rather small values (bubbles). In their case, they studied the probability density function of the solid volume fraction and a limiting value for the dense region of 0.4 was arbitrary selected. Hulme et al., 2005 varied the volume fraction cut off, in a $2 \mathrm{D}$ simulation from 0.15 
to 0.3 , concluding that the definition of the bubble boundary is important as the bubble size changes with the selected threshold.

Fig. 8 illustrates the dependence of the bubble passing frequency (i.e. bubble count) and chord length on the particle volume fraction cut off value. The passing frequency of the detected bubbles was determined by dividing the number of bubbles at a given position by the time duration of the bubble sampling. Thresholds for particle volume fractions, $\alpha_{p, t h}$, covering the ranges of previous studies $(0.2,0.3,0.4)$ are considered. Results of $\alpha_{p, t h}$ equal to the time average value of the whole particle fraction signal (i.e. mean of $\alpha_{p}$ ) are also shown. In Fig. 8a, it can be seen that the number of bubbles counted decreases as lower values of thresholds are used. Fewer bubbles are miscounted using the mean volume fraction than the rest of thresholds values shown in the figure. When using the threshold $\alpha_{p, t h}=0.4$ and the time average particle volume fraction, the bubble frequency starts to decrease at a certain height in the bed. For lower threshold values the bubble count remains almost constant along the bed height.

Fig. 8b shows a growth of bubble size along the bed height, although the rate of increase in the cases of $\alpha_{p, t h}=0.2$ and 0.3 is lower than for higher threshold values. Moreover, the mean bubble pierced length increases as the threshold value increases. This dependence of the bubble pierced length on the threshold used indicates that, as shown by Buyevich et al. (1995), there is not a sudden change from void to emulsion but a region of varying porosity surrounding the bubbles, as it is also reflected by the particle volume fraction signals plotted in Fig. 4d.

In the simulation, when very small bubbles cross the acquisi tion point or the bubbles are laterally pierced, the particle volume fraction level drop is small due to the mentioned gradual decrease of porosity in the bubble boundary and the finite resolution of the computational mesh. Hence, a number of bubbles will not be detected if a low threshold value is used. According to these results, it is confirmed that it is difficult to uniquely define the bubble edge and the threshold selected considerably affects both the bubble count and the deduced chord length.

The time averaged $\alpha_{p}$ has been chosen as the threshold $\alpha_{p, t h}$ in the present work, which is calculated for each acquisition point in the bed. By this way, the threshold is automatically adapted to the particular conditions of each kind of bed or regime. For example, $\alpha_{p, t h}$ can reflect the spatially varying porosity of the emulsion phase encountered in fluidized beds under vigorously bubbling regimes.

\subsubsection{Experimental and simulated bubble pierced length and} velocity

The bubble pierced lengths and velocities at different radial and axial positions in the bed were obtained applying the procedure explained in Section 4.1 and are shown in Fig. 9.
Considering the simulation results first, the signals were recorded at different axial and radial positions for two different azimuth positions $\left(0^{\circ}\right.$ and $\left.120^{\circ}\right)$ in order to verify the degree of axisymmetry of the solution. Differences between these two angular positions are very little and only shown along the radial direction as an example. Therefore, the simulation results of the remaining figures are calculated using the signals from both azimuth positions. By this way, more information for the con struction of the mean quantities is obtained. In Fig. 9, the pierced lengths calculated from the simulated pressure records are almost equal to those from the simulated volume fraction signals, which demonstrates the coherence of the processing methods. Accord ing to Fig. 9, the bubble size and velocity profiles are nearly flat along the radial direction, but they grow with the distance to the distributor as expected if coalescence is considered.

The bubble pierced length measurements from the optical probe signals and the values obtained from particle volume fraction and pressure in the simulation, at different radial positions (Fig. 9a), differ less than $5 \%$ to $10 \%$. In the axis the discrepancy is larger (25\%). Along the bed height (Fig. 9b) the differences found remain almost constant in the range of 10 15\%. Bubble velocities (Fig. 9c and d) calculated from the simulation are larger than the experimental values, the higher discrepancy being around 30\% near the wall $(r / R=0.8)$, but less than $10 \%$ in other positions.

\subsubsection{Experimental and simulated bubble passing frequency}

The number of bubbles detected along the radial and axial directions using the experimental optical probe signals and the simulated signals of pressure and particle volume fraction are compared in Fig. 10.

The three profiles in Fig. 10a present lower values near the wall and the axis. In the experimental case this tendency is more pronounced. Comparing the number of detected bubbles in the simulation, it is observed, as previously reasoned, that the particle count is higher using the particle volume fraction signal than using the differential pressure signal. Previous results shown in Fig. 9, in which mean pierced length and velocity of bubbles calculated from the simulated pressure are in good agreement with the results from particle volume fraction, seem to indicate that the uncounted bubbles using differential pressure signals are equally distributed along all the range of bubble sizes and velocities. Note that the experimentally counted bubbles from the optical signals agree within a $30 \%$ of difference (in the worst case) with the bubble frequency obtained from the simulated pressure.

Fig. 10b shows that the passing frequencies of the detected bubbles using the optical signals and the signals from the simulated differential pressure are almost coincident but lower than the count
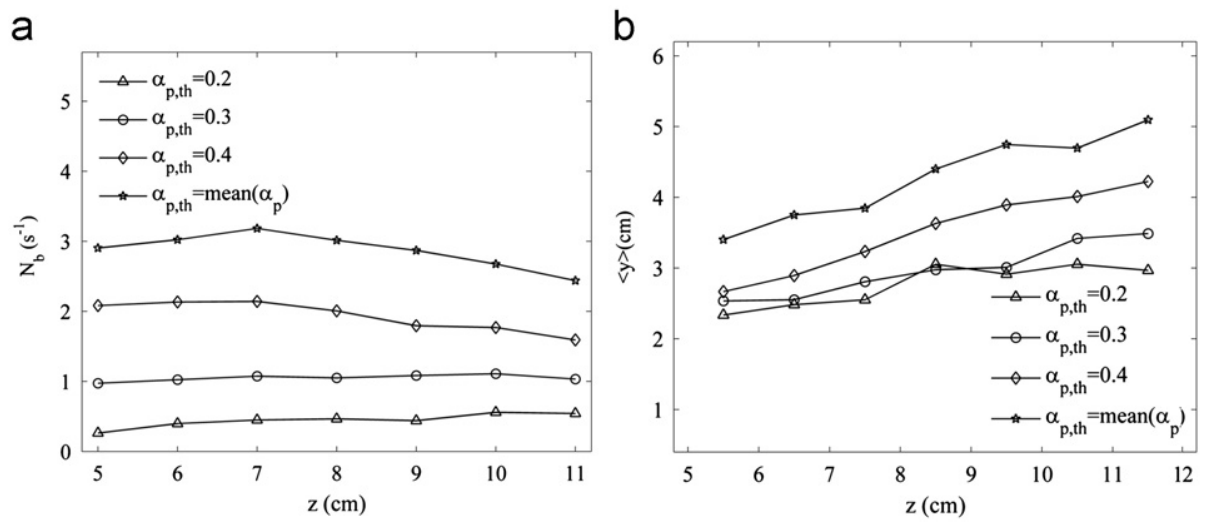

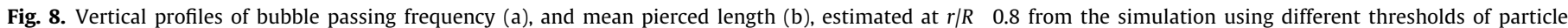
volume fraction during the detection of bubbles. 
a

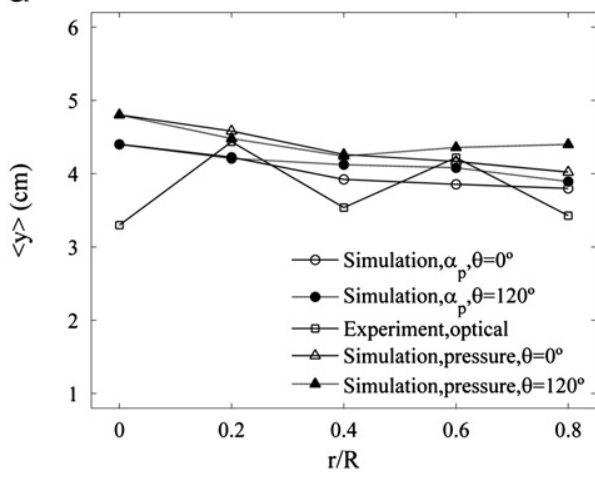

C

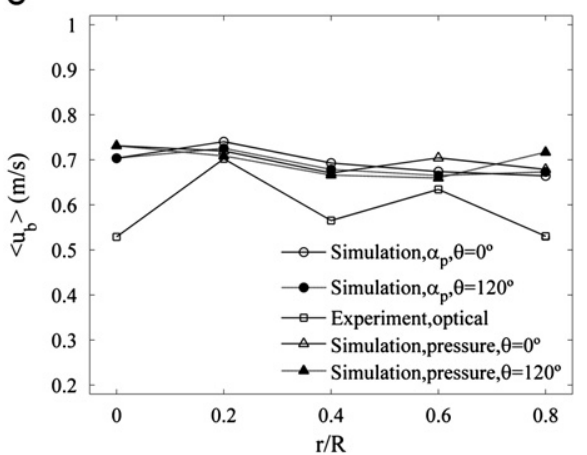

b

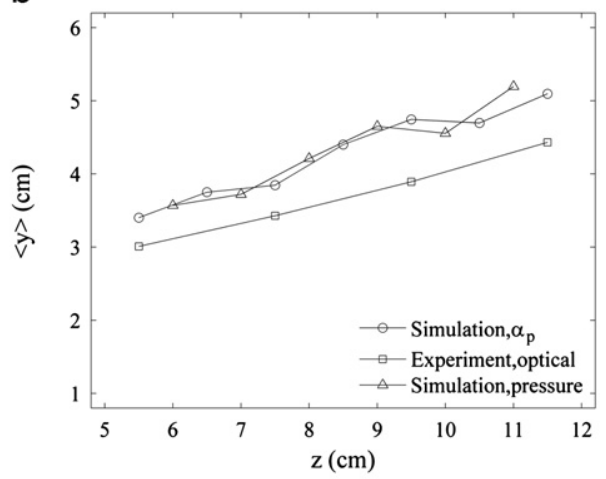

d

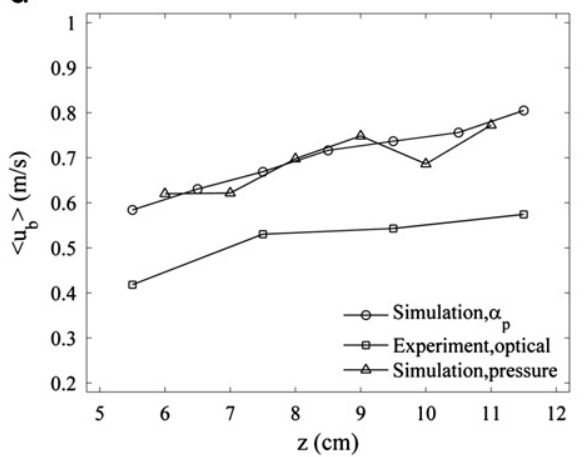

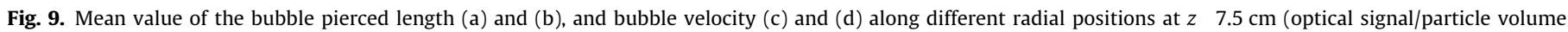
fraction) or $z \quad 8 \mathrm{~cm}$ (differential pressure) and axial positions at $r / R \quad 0.8$.

a

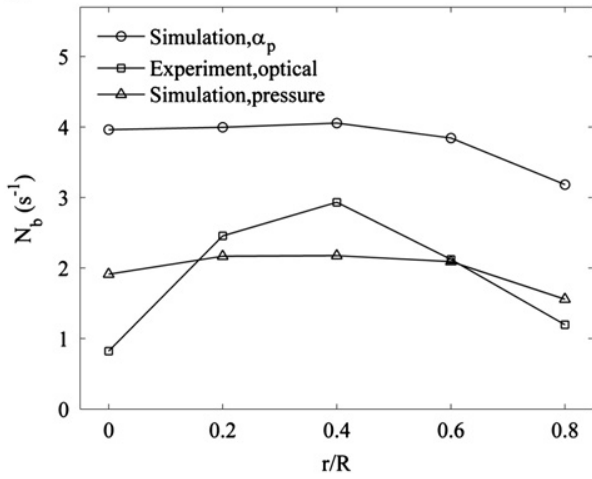

b

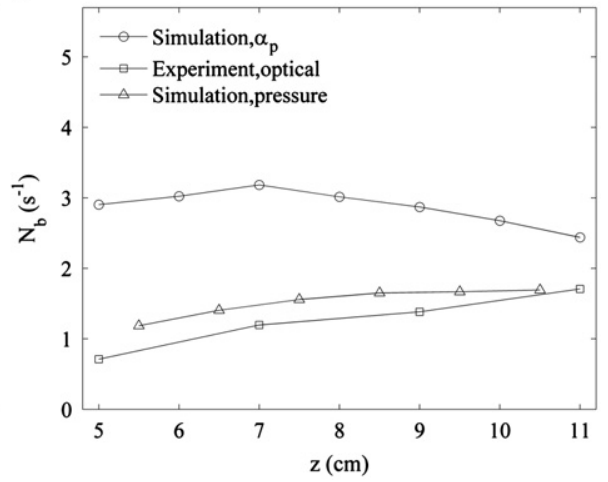

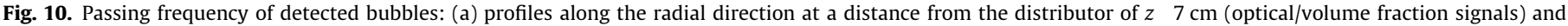
$z \quad 7.5 \mathrm{~cm}$ (differential pressure) and (b) profiles along the axial direction at $r / R \quad 0.8$.

given by the signal from the simulated particle volume fraction. As already stated, the cut off values are more restrictive in the first two signals than in the third one. Nevertheless, the differences tend to decrease as the distance to the distributor increases. This is probably so because at higher positions in the bed, larger bubbles are found (as seen in Fig. 9b) and therefore the threshold effect is less pronounced. The same reasoning may be used to explain the gently growth with height of the bubbles counted with the simulated differential pressure and the optical probes as larger bubbles are more easily detected. Besides, this also occurred in the sensitivity analysis shown in Fig. 8a in which larger threshold values of the simulated particle fraction lead to a decrease of the bubble passing frequency along the bed axis, $z$, whereas small values of the threshold (more restrictive) lead to a slight increase of bubble frequency with $z$. Nonetheless, further experimental results are required to determine whether the bubble passing frequency increases due to additional effects such as the redistribution of the bubbles within the bed.

\subsubsection{Bubble pierced length and diameter distributions}

In Fig. 11 the probability density functions of pierced length and volume equivalent diameter calculated using the maximum entropy method (Santana et al., 2006) over the simulated particle volume fraction and the experimental optical probe signals are shown. The minimum value that was measurable with the probes is limited by their separation, and therefore the obtained density functions are distributions of pierced lengths larger than $1 \mathrm{~cm}$ (Sobrino et al., 2009a). A very similar shape of the distributions is found for the simulated and experimental cases with a wider distribution as long as the measurement point is placed higher in the bed. In Fig. 11a and b, the bubbles with small chord lengths 
a

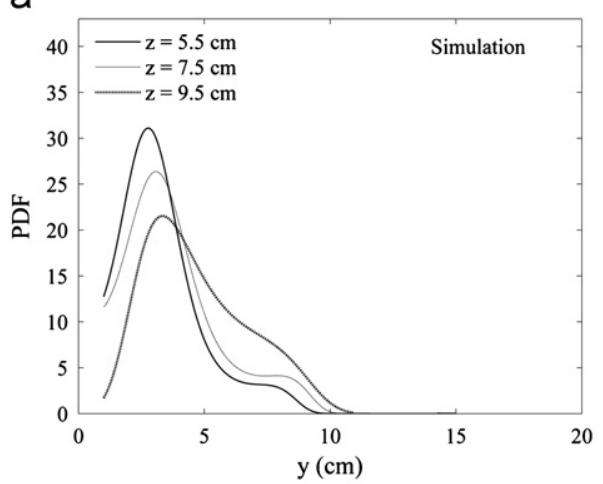

C

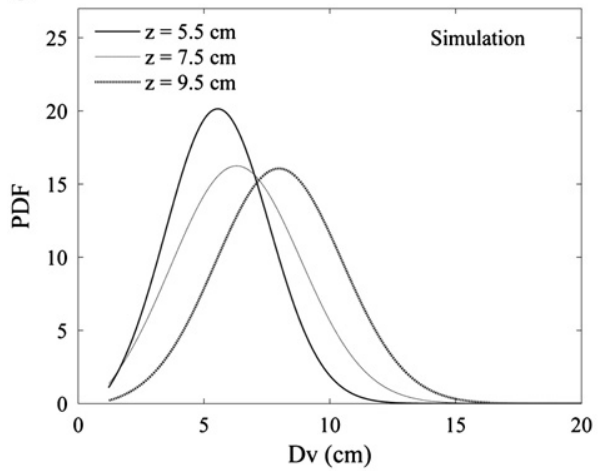

b

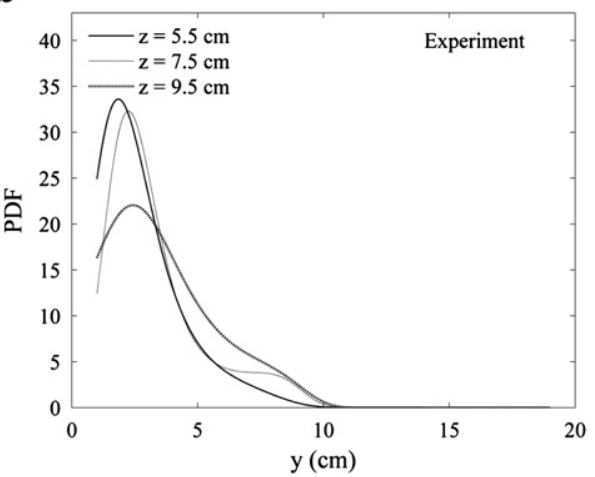

d

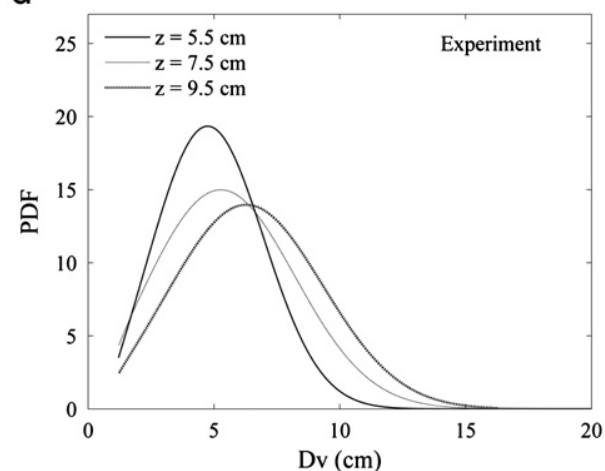

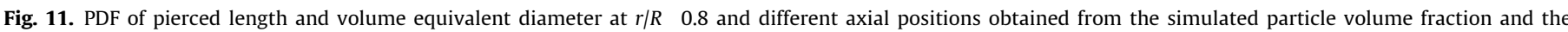
experimental optical probe signals.

tend to disappear from the PDF when $z$ increases, whereas bubbles with large chord lengths increase in number, which reflects the coalescence phenomenon. The statistical modes of the simulated case distributions are around 25\% higher than the experimental ones. It should be recalled that use of MEM to obtain the PDF of bubble pierced length is straightforward as the PDF is constructed directly from the simulated particle volume fraction and the optical signal. On the contrary, the estimation of the bubble diameter distribution (Fig. 11c and d) from pierced length raw moments had to be inferred, as diameters cannot be directly measured. In the present case, as could be seen in Fig. 5, the approximation of the shape of the bubbles to a truncated spheroid employed for this derivation seems to be somehow corroborated by the simulation results, although further quanti tative studies on the bubble shape from the simulated signals are needed.

The mean values of the distributions of bubble volume equivalent diameter shown in Fig. 11c and $d$ are plotted in Fig. 12 against the axial position. The mean bubble diameter increases with height in harmony with the assumption of the progressive coalescence between bubbles. Very good agreement between simulation and experiments is found not only regarding the mean quantities but also the standard deviation of the distributions indicated by the vertical bars in Fig. 12. The rate of increase of the bubble mean diameter with the distance over the distributor in the simulation is similar to the increase obtained from the optical probes in the real bed. The simulated mean diameter is between $10 \%$ and $15 \%$ higher in the simulation than in the experiments. In Fig. 12, the expected diameter curves apply ing Darton et al. (1977) and Argawal (1985) correlations are also depicted. The prediction due to Darton et al. (1977) correlation underestimates both the simulation and experimental bubble diameter in Fig. 12. This is coherent with previously reported results of $3 \mathrm{D}$ simulations (Cammarata et al., 2003). The

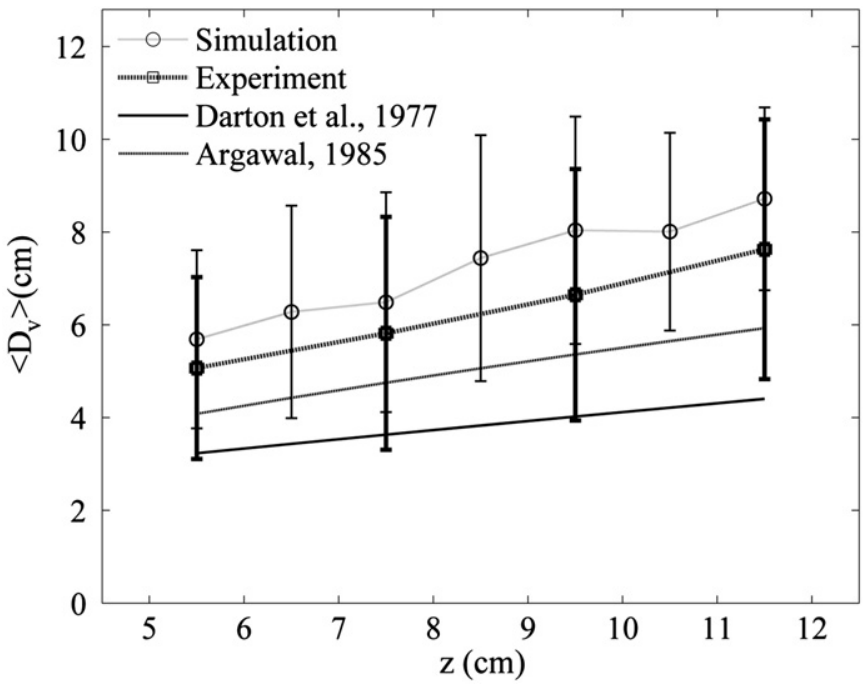

Fig. 12. Mean volume equivalent diameter along the axial position for $r / R \quad 0.8$ obtained from the simulated particle volume fraction and the experimental optical probe signals. The correlation curves due to Darton et al. (1977) and Argawal (1985) are also included. The bars refer to the range due to one standard deviation of the results.

correlation of Argawal (1985) also underestimates the mean bubble diameter but is closer to the simulation and the experi mental results than the model of Darton et al. (1977). Note that both correlations have the same trend than the simulation and the experiment results, and the differences between the Darton et al. (1977) and Argawal (1985) curves are of the same order of the differences between the Argawal (1985) correlation and the curve from the experiments. 


\section{Conclusions}

The bubble behavior in a cylindrical fluidized bed in bubbling regime has been characterized in the present work with the combined aid of experiments and a full $3 \mathrm{D}$ simulation. An Eulerian Eulerian (CFD) two fluid formulation employing well known closure models and standard parameters, with no previous adjustments or parameter tuning, was used for the simulation. Pressure and optical/volume fraction signals obtained from experi mental probes in a real bed and from virtual probes in the simulation were compared and used to describe the essential features of the bubble behavior and the resulting dynamics of the bed.

A main conclusion derived from the comparison between the simulation and the experimental results is that the employed two fluid model is able to reproduce the bubble behavior in the studied bed with reasonable accuracy. In particular, the results demonstrate that the experiments and the simulation provide a similar pattern in the power spectra of pressure. This similarity was also observed in the spectra of optical/volume fraction. Besides, the same frequency shift in the differential pressure spectra, compared to the absolute pressure spectra, was found in the experiments and in the simulation. The spectral analysis also showed that the differential pressure spectrum is clearly related to the particle fraction spectrum, confirming the local character of the information provided by differential pressure probes. At this regard, it seems crucial the use of a fully $3 \mathrm{D}$ simulation, as the bubble coalescence and interaction with the free surface of the fluidized bed are eminently three dimensional phenomena. The experimental radial profiles of bubble pierced length and bubble velocity calculated from optical probes, were in good agreement with the results calculated from the simulated signals of differential pressure and volume fraction. The larger differences were around $25 \%$ for the pierced length and $30 \%$ for the bubble velocity, the simulated values being higher than the experimental ones. These differences may be attributed to several causes, including the inter ference of the optical probe in the real bed and the not totally equivalent nature of scattered light and volume fraction signals.

Another important conclusion, stemming from the analysis of the bubble pierce length and passing frequency, is that the selection of the bubble detection threshold has an impact in the simulation results that cannot be obviated. The soft transition between bubbles and dense phase in the volume fraction signals from the simulation makes difficult to uniquely define the bubble edge. In the present work, the time average particle volume fraction is proposed as threshold value for the detection of the simulated bubbles. For the studied bed, this particular threshold ensured that the bubble pierced lengths and velocities obtained from the simulated volume fraction matched those obtained from the simulated differential pressure.

It is also worth mentioning that the maximum entropy method, used in this work for the estimation of the probability density function (PDF) of bubble pierced length and bubble diameter, seems to corroborate the consistency of the simulated particle volume fraction with the experimental optical signal. Both the experiments and the simulation revealed that the density functions of pierced length, and hence those of bubble diameter, become wider as the distance to the distributor increases. Besides, the mean bubble diameter also increases with the distance to the distributor. The experiments and the simulation provide very similar rates of bubble growth, although the size of the simulated bubbles was higher (up to $20 \%$ ) than the size obtained from the experiments.

Despite the promising results here presented, further experi mental information together with a dedicated series of simulations would be required to gain precise knowledge of the sources of the discrepancies observed between some of the experimental and simulation results.

\section{Nomenclature}

$d_{p} \quad$ particle diameter $(\mathrm{m})$

$D_{v} \quad$ bubble volume equivalent diameter $(\mathrm{m})$

$e_{p p} \quad$ restitution coefficient (dimensionless)

$f_{e} \quad$ theoretical natural frequency of the bed $(\mathrm{Hz})$

$f_{d} \quad$ dominant frequency of the pressure spectrum $(\mathrm{Hz})$

$f_{\text {dif }} \quad$ characteristic frequency of the differential pressure spectrum $(\mathrm{Hz})$

$f_{o p} \quad$ characteristic frequency of the optical/particle fraction spectrum $(\mathrm{Hz})$

g gravity acceleration $\left(9.81 \mathrm{~m} / \mathrm{s}^{2}\right)$

$h_{0} \quad$ static bed height $(\mathrm{m})$

$\mathrm{H}$ total height of the column (m)

I unit tensor (dimensionless)

$k_{\Theta} \quad$ granular temperature diffusion coefficient $(\mathrm{kg} / \mathrm{m} \mathrm{s})$

$K_{g p} \quad$ gas particle momentum exchange coefficient $\left(\mathrm{kg} / \mathrm{m}^{3} \mathrm{~s}\right)$

$N_{b} \quad$ number of bubbles per unit time $\left(\mathrm{s}^{1}\right)$

$N_{i} \quad$ number of iterations per time step

$p \quad$ static pressure (Pa)

$r / R \quad$ dimensionless radial position

$R \quad$ bed internal radius (m)

$t$ time $(\mathrm{s})$

$u_{b} \quad$ bubble velocity $(\mathrm{m} / \mathrm{s})$

$U_{g} \quad$ superficial gas velocity $(\mathrm{m} / \mathrm{s})$

$U_{m f} \quad$ minimum fluidization velocity $(\mathrm{m} / \mathrm{s})$

v velocity vector $(\mathrm{m} / \mathrm{s})$

$y \quad$ bubble pierced length ( $\mathrm{m}$ )

$z \quad$ vertical distance to the distributor $(\mathrm{m})$

\section{Greek letters}

$\begin{array}{ll}\alpha & \text { volume fraction } \\ \Delta t & \text { time step (s) } \\ \Delta t_{i / o / S} & \text { offset times between signals (s) } \\ \gamma_{\Theta} & \text { collisional dissipation energy }\left(\mathrm{kg} / \mathrm{m} \mathrm{s}^{3}\right) \\ \lambda & \text { bulk viscosity (Pa s) } \\ \mu & \text { shear viscosity (Pa s) } \\ \theta & \text { azimuth angle (deg.) } \\ \Theta & \text { granular temperature }\left(\mathrm{m}^{2} / \mathrm{s}^{2}\right) \\ \rho & \text { density }\left(\mathrm{kg} / \mathrm{m}^{3}\right) \\ \tau & \text { shear stress tensor }(\mathrm{Pa})\end{array}$

\section{Subscripts}

$\begin{array}{ll}\text { col } & \text { collisional } \\ \text { dif } & \text { differential } \\ \text { fr } & \text { frictional } \\ g & \text { gas phase } \\ \text { kin } & \text { kinematic } \\ \text { max } & \text { maximum } \\ \text { op } & \text { optical } \\ p & \text { particle phase } \\ \text { th } & \text { threshold }\end{array}$

\section{Acknowledgments}

This work has been partially funded by the Spanish Govern ment (Project DPI2009 10518) and the Autonomous Community of Madrid (Project S2009/ENE 1660). The authors gratefully appreciate their support.

\section{References}

Argawal, P.K., 1985. Bubble characteristics in gas-fluidized beds. Chemical Engineering Research and Design 63, 323-337. 
Ahuja, G.N., Patwardhan, A.W., 2008. CFD and experimental studies of solids holdup distribution and circulation patterns in gas-solid fluidized beds. Chemical Engineering Journal 143, 147-160.

Baskakov, A.P., Tuponogov, V.G., Filippovsky, N.F., 1986. A study of pressure fluctuations in a bubbling fluidized bed. Powder Technology 45, 113-117.

Bi, H.T., 2007. A critical review of the complex pressure fluctuation phenomenon in gas-solids fluidized beds. Chemical Engineering Science 62, 3473-3493.

Buyevich, Y.A., Yates, J.G., Cheesman, D.J., Wu, K.-T., 1995. A model for the distribution of voidage around bubbles in a fluidized bed. Chemical Engineering Science 50, 3155-3162.

Cammarata, L., Lettieri, P., Micale, G.D.M., Colman, D., 2003. 2D and 3D CFD simulations of bubbling fluidized beds using Eulerian-Eulerian models. International Journal of Chemical Reactor Engineering 1 ArtA48.

Darton, R.C., LaNauze, R.D., Davidson, J.F., Harrison, D., 1977. Bubble growth due to coalescense in fluidised beds. Transactions of the Institution of Chemical Engineers 55, 274-280.

Davidson, J.F., Harrison, D., 1963. Fluidised Particles. Cambridge University Press, Cambridge.

Deen, N.G., Van Sint Annaland, M., Van der Hoef, M.A., Kuipers, J.A.M., 2007. Review of discrete particle modelling of fluidized beds. Chemical Engineering Science 62, 28-44.

Dent, D., LaNauze, R.D., Joyce, T., Fulford, V., Peeler, P., 1989. Differential pressure measurements - their application to measurement of fluidized bed combustion parameters. In: Proceedings of the International Conference on Fluidized Bed Combustion, Published by the American Society of Mechanical Engineers (ASME), New York, NY, United States, pp. 451-456.

Fluent Inc., 2006. Fluent 6.3 User's Guide. http://www.fluent.com.

Gidaspow, D., 1994. Multiphase Flow and Fluidization. Academic Press, Boston.

Gidaspow, D., Bezburuah, R., Ding, J., 1992. Hydrodynamics of circulating fluidized beds, kinetic theory approach. In: Fluidization VII, Proceedings of the 7th Engineering Foundation Conference on Fluidization, pp. 75-82.

Goldschmidt, M.J.V., Kuipers, J.A., van Swaaij, W.P.M., 2001. Hydrodynamic modelling of dense gas-fluidised beds using the kinetic theory of granular flow: effect of coefficient of restitution on bed dynamics. Chemical Engineering Science 56, 571-578.

Grace, J.R., Taghipour, F., 2004. Verification and validation of CFD models and dynamic similarity for fluidized beds. Powder Technology 139, 99-110.

Hutchinson, B.R., Raithby, G.D., 1986. A multigrid method based on the additive correction strategy. Numerical Heat Transfer 9, 511-537.

Hulme, I., Clavell, E., van der Lee, L., Kantzas, A., 2005. CFD modeling and validation of bubble propierties for a bubbling fluidized bed. Industrial \& Engineering Chemical Research 44, 4254-4266.

Johansson, K., van Wachem, B.G.M., Almstedt, A.E., 2006. Experimental validation of CFD models for fluidized beds: influence of particle stress models, gas phase compressibility and air inflow models. Chemical Engineering Science 61, 1705-1717.

Johnsson, F., Zijerveld, R.C., Schouten, J.C., van den Bleek, C.M., Leckner, B., 2000. Characterization of fluidization regimes by time-series analysis of pressure fluctuations. International Journal of Multiphase Flow 26, 663-715.

Ladd, A.J.C., Verberg, R., 2001. Lattice-Boltzmann simulations of particle fluid suspensions. Journal of Statistical Physics 104, 1191-1251.

Liu, J.Z., Grace, J.R., Bi, X.T., 2003. Novel multifunctional optical-fiber probe: I. Development and validation. A.I.Ch.E. Journal 49, 1405-1420.

Liu, M., Zhang, Y., Bi, H., Grace, J.R., Zhu, Y., 2010. Non-intrusive determination of bubble size in gas-solid fluidized bed: an evaluation. Chemical Engineering Science 65, 3485-3493.

Lun, C.K.K., Savage, S.B., Jeffrey, D.J., Chepurniy, N., 1984. Kinetic theories for granular flow: inelastic particles in couette flow and slightly inelastic particles in a general flow field. Journal of Fluid Mechanics 140, 223-256.
McKeen, T., Pugsley, T., 2003. Simulation and experimental validation of a freely bubbling bed of FCC catalyst. Powder Technology 129, 139-152.

Passalacqua, A., Marmo, L., 2009. A critical comparison of frictional stress models applied to the simulation of bubbling fluidized beds. Chemical Engineering Science 160, 2795-2806.

Patankar, S.V., 1980. Numerical Heat Transfer and Fluid Flow. Taylor \& Francis.

Patil, D.J., van Sint Annaland, M., Kuipers, J.A.M., 2005. Critical comparison of hydrodynamic models for gas-solid fluidized beds-Part II: freely bubbling gas-solid fluidized beds. Chemical Engineering Science 60, 73-84.

Peirano, E., Delloume, V., Leckner, B., 2001. Two- or three-dimensional simulations of turbulent gas-solid flows applied to fluidization. Chemical Engineering Science 56, 4787-4799.

Peirano, E., Delloume, V., Johnsson, F., Leckner, B., Simonin, O., 2002. Numerica simulation of the fluid dynamics of a freely bubbling fluidized bed: influence of the air supply system. Powder Technology 122, 69-82.

Ramayya, A.V., Venkateshan, S.P., Kolar, A.K., 1996. Estimation of bubble parameters from differential pressure measurements in gas-fluidized beds. Powder Technology 87, 113-126.

Rowe, P.N., Masson, H., 1981. Interaction of bubbles with probes in gas fluidized beds. Transactions of the Institution of Chemical Engineering 59, 117-185.

Roy, R., Davidson, J.F., Tupogonov, V.G., 1990. The velocity of sound in fluidised beds. Chemical Engineering Science 45, 3233-3245.

Santana, D., Rodríguez-Rodríguez, J., Almedros-Ibáñez, J.A., Martínez-Bazán, C., 2006. Characteristics lengths and maximum entropy estimation from probe signals in the ellipsoidal bubble regime. International Journal of Multiphase Flow 32, 1123-1139.

Schaeffer, D.G., 1987. Instability in the evolution equations describing incompressible granular flow. Journal of Differential Equations 66, 19-50.

Schweitzer, J.-M., Bayle, J., Gauthier, T., 2001. Local gas hold-up measurements in fluidized bed and slurry bubble column. Chemical Engineering Science 56, 1103-1110.

Sobrino, C., Almendros-Ibáñez, J.A., Santana, D., Vázquez, C., de Vega, M., 2009a. Maximum entropy estimation of the bubble size distribution in fluidized beds. Chemical Engineering Science 64, 2307-2319.

Sobrino, C., Acosta-Iborra, A., Santana, D., de Vega, M., 2009b. Bubble characteristics in a bubbling fluidized bed with a rotating distributor. Internationa Journal of Multiphase Flow 35, 970-976.

Taghipour, F., Ellis, N., Wong, C., 2005. Experimental and computacional study of gassolid fluidized bed hydrodynamics. Chemical Engineering Science 60, 6857-6867.

van Wachem, B.G.M., Schouten, J.C., Krishna, R., van den Bleek, C.M., 1998. Eulerian simulations of bubbling behaviour in gas-solid fluidised beds. Computers \& Chemical Engieering 22, 299-306.

van Wachem, B.G.M., Schouten, J.C., Krishna, R., van den Bleek, C.M., 1999. Validation of the Eulerian simulated dynamic behaviour of gas-solid fluidised beds. Chemical Engineering Science 54, 2141-2149.

van Wachem, B.G.M., Almstedt, A.E., 2003. Methods for multiphase computational fluid dynamics. Chemical Engineering Journal 96, 81-98.

Wang, S., Lu, H., Li, X., Yu, L., Ding, J., Zhao, Y., 2008. CFD simulations of bubbling beds of rough spheres. Chemical Engineering Science 63, 5653-5662.

Wang, J., van der Hoef, M.A., Kuipers, J.A.M., 2010. Coarse grid simulation of bed expansion characteristics of industrial-scale gas-solid bubbling fluidized beds. Chemical Engineering Science 65, 2125-2131.

Werther, J., 1974. Bubbles in gas fluidised beds-part I. Transactions of the Institution of Chemical Engineers 52, 149-159.

Xie, N., Battaglia, F., Pannala, S., 2008. Effects of using two- versus threedimensional computational modeling of fluidized beds. Part I, hydrodynamics. Powder Technology 182, 1-13. 